\title{
The Existence of the Sign-Changing Solutions for the Kirchhoff-Schrödinger-Poisson System in Bounded Domains
}

\author{
Cun-bin An $\mathbb{D}^{1},{ }^{1}$ Jiangyan Yao, ${ }^{2}$ and Wei Han $\mathbb{D}^{2}$ \\ ${ }^{1}$ School of Mathematics and Statistics, Shanxi Datong University, Datong, Shanxi 037009, China \\ ${ }^{2}$ Department of Mathematics, North University of China, Taiyuan, Shanxi 030051, China
}

Correspondence should be addressed to Cun-bin An; dt_ancunbin@126.com

Received 6 September 2019; Accepted 18 March 2020; Published 19 May 2020

Academic Editor: Phuc Phung-Van

Copyright (c) 2020 Cun-bin An et al. This is an open access article distributed under the Creative Commons Attribution License, which permits unrestricted use, distribution, and reproduction in any medium, provided the original work is properly cited.

In this paper, we study a class of the Kirchhoff-Schrödinger-Poisson system. By using the quantitative deformation lemma and degree theory, the existence result of the least energy sign-changing solution $u_{0}$ is obtained. Meanwhile, the energy doubling property is proved, that is, we prove that the energy of any sign-changing solution is strictly larger than twice that of the least energy. Moreover, we also get the convergence properties of $u_{0}$ as the parameters $b \searrow 0$ and $\lambda \searrow 0$.

\section{Introduction and the Main Results}

In this paper, the following Kirchhoff-Schrödinger-Poisson system is considered:

$$
\begin{cases}-\left(a+b \int_{\Omega}|\nabla u|^{2} d x\right) \Delta u+\lambda \phi x=g(u), & x \in \Omega \\ -\Delta \phi=u^{2}, & x \in \Omega, \\ u=\phi=0, & x \in \partial \Omega,\end{cases}
$$

where $\Omega \subset \mathbb{R}^{3}$ is a bounded domain with a smooth boundary $\partial \Omega, a, b, \lambda \in \mathbb{R}^{+}=(0,+\infty)$, and $g \in C(\mathbb{R}, \mathbb{R})$ satisfies some basic assumptions.

For $b=0$, problem (1) reduces to the following Schrödinger-Poisson system:

$$
\left\{\begin{array}{l}
-a \Delta u+\lambda \phi(x) u=g(u), x \in \Omega, \\
-\Delta \phi=u^{2}, x \in \Omega \\
\phi, u=0, x \in \partial \Omega
\end{array}\right.
$$

Alves and Souto [1] studied the above SchrödingerPoisson system for $a=\lambda=1$. Under some suitable assumptions on the nonlinearity $g(u)$, by using the deformation lemma and Brouwer's topological degree theory, they proved that the above system possessed a least-energy sign-changing solution, which changed sign only once.

For $\lambda=0$, the problem (1) reduces to the following problem:

$$
\begin{cases}-\left(a+b \int_{\Omega}|\nabla u|^{2} d x\right) \Delta u=g(u), & x \in \Omega \\ u=0, & x \in \partial \Omega .\end{cases}
$$

The problem (3) has been studied in [2, 3]. Under different assumptions on $g(u)$, the authors in [2,3] obtained the existence and some qualitative properties of the signchanging solution by using the Non-Nehari manifold method and deformation lemma. We can find that the results in [3] improve and generalize the results in [2]. In fact, the studies about the existence of the positive solutions, signchanging solutions for a class of elliptic equations, have been studied extensively. For more details about such problems, we refer the reader to [4-19].

To our best knowledge, the results of the sign-changing solutions for the Kirchhoff-Schrödinger-Poisson system under a weak assumption that $g \in C(\mathbb{R}, \mathbb{R})$ have not been studied yet. This paper attempts to fill this gap in the literature. Motivated by the above papers, we study the problem 
(1). For the purpose of getting the results, we use the variational method and some mathematical skills to obtain the existence of the sign-changing solution and its corresponding properties.

In this paper, we assume $g \in C(\mathbb{R}, \mathbb{R})$ satisfies the following four conditions:

$$
\begin{aligned}
& \left(g_{1}\right) \lim _{s \rightarrow 0}(g(s) / s)=0 \\
& \left(g_{2}\right) \lim _{|s| \rightarrow \infty} g(s) / s^{3}=\infty \\
& \left(g_{3}\right)|g(s)|<c_{0}\left(1+|s|^{4}\right), \forall s \in \mathbb{R} \text {, where } c_{0} \text { is a constant } \\
& \left(g_{4}\right) \text { there exists a } \theta_{0} \in(0,1) \text { such that for any } s>0 \text { and } \\
& \mathbb{R} \backslash\{0\}, \\
& {\left[\frac{g(\tau)}{\tau^{3}}-\frac{g(s \tau)}{(s \tau)^{3}}\right] \operatorname{sign}(1-s)+\frac{a \theta_{0} \lambda_{1}\left|1-s^{2}\right|}{(s \tau)^{2}} \geq 0}
\end{aligned}
$$
$\tau \in \mathbb{R} \backslash\{0\}$,

where $\lambda_{1}$ is the first eigenvalue for the following problem:

$$
\begin{cases}-\Delta u=\lambda u, & x \in \Omega, \\ u=0, & x \in \partial \Omega .\end{cases}
$$

Throughout this paper, we will use the following notations.

Let $H=H_{0}^{1}(\Omega)$ be the usual Sobolev space equipped with the following norm:

$$
\|u\|=\left(\int_{\Omega}|\nabla u|^{2} d x\right)^{1 / 2}
$$

The usual $L^{p}$ norm is denoted by $\|u\|_{p}=\left(\int_{\Omega}|u|^{p} d x\right)^{1 / p}$. In this way, we know $\|u\|=\|\nabla u\|_{2}$.

For the Poisson system,

$$
\begin{cases}-\Delta \phi=u^{2}, & x \in \Omega, \\ \phi=0, & x \in \partial \Omega,\end{cases}
$$

where there exists a unique $\phi_{u}=1 / 4 \pi \int_{\Omega}\left(u^{2}(y) /|x-y|\right)$ $d y \in H$, such that $\phi_{u}$ satisfies the above system. It is known that $\phi_{u}$ satisfies the following conditions [17-19]:

(i) $\int_{\Omega} \phi_{u} u^{2} d x=\int_{\Omega}\left|\nabla \phi_{u}\right|^{2} d x \leq C\|\nabla u\|_{2}^{4}$

(ii) $\phi_{u} \geq 0$ and $\phi_{u}>0$ for $u \neq 0$

(iii) for $u=u^{+}+u^{-} \in H, \phi_{u^{+}+u^{-}}=\phi_{u^{+}}+\phi_{u^{-}}$and for $t \neq 0$, $\phi_{t u}=t^{2} \phi_{u}$

(iv) if $u_{n} \rightarrow u$ in $H_{0}^{1}(\Omega)$, then $\phi_{u_{n}} \rightarrow \phi_{u}$ in $H_{0}^{1}(\Omega)$ and $\lim _{n \rightarrow \infty} \int_{\Omega} \phi_{u_{n}} u_{n}^{2} d x=\int_{\Omega} \phi_{u} u^{2} d x$; if $u_{n}^{ \pm} \rightarrow u^{ \pm}$in $H_{0}^{1}$ $(\Omega)$, then $\liminf _{n \rightarrow \infty} \int_{\Omega} \phi_{u_{n}}\left(u_{n}^{ \pm}\right)^{2} d x=\int_{\Omega} \phi_{u}\left(u^{ \pm}\right)^{2} d x$.

Consequently, $(u, \phi) \in H_{0}^{1}(\Omega) \times H_{0}^{1}(\Omega)$ is a solution of problem (1), that is, $\phi=\phi_{u}$ and $u \in H_{0}^{1}(\Omega)$ are a solution of the following problem:

$$
\begin{cases}-\left(a+b \int_{\Omega}|\nabla u|^{2} d x\right) \Delta u+\lambda \phi_{u} u=g(u), & x \in \Omega, \\ u=0, & x \in \partial \Omega .\end{cases}
$$

In this paper, $u \in H_{0}^{1}(\Omega)$ is called a solution for problem (1), which implies $\left(u, \phi_{u}\right) \in H_{0}^{1}(\Omega) \times H_{0}^{1}(\Omega)$ is a solution of problem (1).

Next, we can define the energy functional corresponding to problem (1) $F: H_{0}^{1}(\Omega) \longrightarrow \mathbb{R}$ by

$$
\begin{aligned}
F(u)= & \frac{a}{2} \int_{\Omega}|\nabla u|^{2} d x+\frac{b}{4}\left(\int_{\Omega}|\nabla u|^{2} d x\right)^{2}+\frac{\lambda}{4} \int_{\Omega} \phi_{u} u^{2} d x \\
& -\int_{\Omega} G(u) d x
\end{aligned}
$$

where $G(s)=\int_{0}^{s} g(t) d t$. Obviously, the functional $F$ is well defined and belongs to $C^{1}(H, \mathbb{R})$. By a simple computation, we have that for any $u, \varphi \in H_{0}^{1}(\Omega)$,

$$
\begin{aligned}
\left\langle F^{\prime}(u), \varphi\right\rangle= & \int_{\Omega} a \nabla u \nabla \varphi d x+b \int_{\Omega}|\nabla u|^{2} d x \int_{\Omega} \nabla u \nabla \varphi d x \\
& +\int_{\Omega} \lambda \phi_{u} u \varphi d x-\int_{\Omega} g(u) \varphi d x .
\end{aligned}
$$

It is clear that the critical points of $F$ are the weak solutions for the problem (1). If $u \in H$ is called a signchanging solution of problem (1), then $u^{ \pm} \neq 0$ and for any $\varphi \in H,\left\langle F^{\prime}(u), \varphi\right\rangle=0$, where $u^{+}(x)=\max \{u(x), 0\}, u^{-}(x)=$ $\min \{u(x), 0\}$.

For $u \in H$ and $u=u^{+}+u^{-}$, by (9) and (10), we have

$$
\begin{aligned}
F(u)= & F\left(u^{+}\right)+F\left(u^{-}\right)+\frac{b}{2}\left\|\nabla u^{+}\right\|_{2}^{2}\left\|\nabla u^{-}\right\|_{2}^{2} \\
& +\frac{\lambda}{4} \int_{\Omega} \phi_{u^{+}}\left(u^{-}\right)^{2} d x+\frac{\lambda}{4} \int_{\Omega} \phi_{u^{-}}\left(u^{+}\right)^{2} d x \\
\left\langle F^{\prime}(u), u^{+}\right\rangle= & \left\langle F^{\prime}\left(u^{+}\right), u^{+}\right\rangle+b\left\|\nabla u^{+}\right\|_{2}^{2}\left\|\nabla u^{-}\right\|_{2}^{2} \\
& +\int_{\Omega} \lambda \phi_{u^{-}}\left(u^{+}\right)^{2} d x \\
\left\langle F^{\prime}(u), u^{-}\right\rangle= & \left\langle F^{\prime}\left(u^{-}\right), u^{-}\right\rangle+b\left\|\nabla u^{+}\right\|_{2}^{2}\left\|\nabla u^{-}\right\|_{2}^{2} \\
& +\int_{\Omega} \lambda \phi_{u^{+}}\left(u^{-}\right)^{2} d x .
\end{aligned}
$$


To get the main results, we restrict $u$ in the following sets:

$$
\begin{aligned}
& \mathscr{M}=\left\{u \in H: u^{ \pm} \neq 0,\left\langle F^{\prime}(u), u^{+}\right\rangle=0,\left\langle F^{\prime}(u), u^{-}\right\rangle=0\right\}, \\
& \mathscr{N}=\left\{u \in H: u \neq 0,\left\langle F^{\prime}(u), u\right\rangle=0\right\} .
\end{aligned}
$$

To get the energy doubling property, we define $m=$ $\inf _{u \in \mathscr{M}} F(u)$ and $c=\inf _{u \in \mathcal{N}} F(u)$.

To prove the convergence property, we give the following definitions. Firstly, we define the energy functional corresponding to (2) $F_{b_{0}}: H_{0}^{1}(\Omega) \longrightarrow \mathbb{R}$ by

$$
F_{b_{0}}(u)=\frac{a}{2} \int_{\Omega}|\nabla u|^{2} d x+\frac{\lambda}{4} \int_{\Omega} \phi_{u} u^{2} d x-\int_{\Omega} G(u) d x .
$$

Similarly, we have

$$
\left\langle F_{b_{0}}{ }^{\prime}(u), \varphi\right\rangle=\int_{\Omega} a \nabla u \nabla \varphi d x+\int_{\Omega} \lambda \phi_{u} u \varphi d x-\int_{\Omega} g(u) \varphi d x .
$$

The set $\mathscr{M}_{b_{0}}$ is defined by $\mathscr{M}_{b_{0}}=\left\{u \in H: u^{ \pm} \neq 0,\left\langle F_{b_{0}}{ }^{\prime}\right.\right.$ $\left.\left.(u), u^{+}\right\rangle=\left\langle F_{b_{0}}{ }^{\prime}(u), u^{-}\right\rangle=0\right\}$.

The energy functional $F_{\lambda_{0}}: H_{0}^{1}(\Omega) \longrightarrow \mathbb{R}$ corresponding to (3) can be defined by

$$
F_{\lambda_{0}}(u)=\frac{a}{2} \int_{\Omega}|\nabla u|^{2} d x+\frac{b}{4}\left(\int_{\Omega}|\nabla u|^{2} d x\right)^{2}-\int_{\Omega} G(u) d x .
$$

Also, we can compute that

$$
\begin{aligned}
\left\langle F_{\lambda_{0}}{ }^{\prime}(u), \varphi\right\rangle= & \int_{\Omega} a \nabla u \nabla \varphi d x+b \int_{\Omega}|\nabla u|^{2} d x \int_{\Omega} \nabla u \nabla \varphi d x \\
& -\int_{\Omega} g(u) \varphi d x .
\end{aligned}
$$

To seek the sign-changing solution of (3), we define the set

$$
\mathscr{M}_{\lambda_{0}}=\left\{u \in H: u^{ \pm} \neq 0,\left\langle F_{\lambda_{0}}{ }^{\prime}(u), u^{+}\right\rangle=\left\langle F_{\lambda_{0}}{ }^{\prime}(u), u^{-}\right\rangle=0\right\} .
$$

The main results of this paper are described as follows.

Theorem 1. Assume that $\left(g_{1}\right)-\left(g_{4}\right)$ hold, then problem (1) possesses a least-energy sign-changing solution $u_{0} \in \mathscr{M}$ such that $F\left(u_{0}\right)=\inf _{M} F>0$, which changes sign only once.

Theorem 2. Assume that $\left(g_{1}\right)-\left(g_{4}\right)$ hold. Then problem (1) possesses a solution $u_{1} \in \mathcal{N}$ such that $F\left(u_{1}\right)=\inf _{\mathcal{N}} F$. Moreover, $m>2 c$.
Theorem 3. Assume that $\left(g_{1}\right)-\left(g_{4}\right)$ hold. Then problem (2) possesses a sign-changing solution $v_{0} \in \mathscr{M}_{b_{0}}$ such that $F_{b_{0}}\left(v_{0}\right)=\inf _{\mathscr{M}_{b_{0}}} F_{b_{0}}>0$, which changes sign only once. Moreover, for any sequence $\left\{b_{n}\right\}$ with $b_{n} \backslash 0$ as $n \longrightarrow \infty$, there exists a subsequence of $\left\{u_{b_{n}}\right\}$, still denoted by $\left\{u_{b_{n}}\right\}$, such that $u_{b_{n}} \longrightarrow u_{b_{0}}$ in $H_{0}^{1}(\Omega)$, where $u_{b_{0}} \in \mathscr{M}_{b_{0}}$ is a sign-changing solution of problem (2) with $F_{b_{0}}\left(u_{b_{0}}\right)=\inf _{\mathscr{M}_{b_{0}}} F_{b_{0}}>0$.

Theorem 4. Assume that $\left(g_{1}\right)-\left(g_{4}\right)$ hold. Then problem (3) has a sign-changing solution $w_{0} \in \mathscr{M}_{\lambda_{0}}$ such that $F_{\lambda_{0}}\left(w_{0}\right)=$ $\inf {\mathscr{M}_{\lambda_{0}}} F_{\lambda_{0}}>0$, which changes sign only once. Moreover, for any sequence $\left\{\lambda_{n}\right\}$ with $\lambda_{n} \searrow 0$ as $n \longrightarrow \infty$, there exists a subsequence of $\left\{u_{\lambda_{n}}\right\}$, still denoted by $\left\{u_{\lambda_{n}}\right\}$, such that $u_{\lambda_{n}} \longrightarrow$ $u_{\lambda_{0}}$ in $H_{0}^{1}(\Omega)$, where $u_{\lambda_{0}} \in \mathscr{M}_{\lambda_{0}}$ is a sign-changing solution of (3) with $F_{\lambda_{0}}\left(u_{\lambda_{0}}\right)=\inf _{\mathscr{M}_{\lambda_{0}}} F_{\lambda_{0}}>0$.

The rest of the paper is organized as follows. In Section 2, we will give several estimates. In Section 3, some critical lemmas are proved. In Section 4, we will give the proof of the existence of the least-energy sign-changing solution. In section 5, the energy doubling property is proved. Section 6 is devoted to proving the convergence property.

\section{Several Estimates}

Lemma 5. If the assumptions $\left(g_{1}\right)-\left(g_{4}\right)$ hold, then

$$
\begin{aligned}
F(u) \geq & F\left(s u^{+}+t u^{-}\right)+\frac{1-s^{4}}{4}\left\langle F^{\prime}(u), u^{+}\right\rangle \\
& +\frac{1-t^{4}}{4}\left\langle F^{\prime}(u), u^{-}\right\rangle \\
& +\frac{a\left(1-s^{2}\right)^{2}\left(1-\theta_{0}\right)}{4}\left\|\nabla u^{+}\right\|_{2}^{2} \\
& +\frac{a\left(1-t^{2}\right)^{2}\left(1-\theta_{0}\right)}{4}\left\|\nabla u^{-}\right\|_{2}^{2} \\
& +\frac{b\left(s^{2}-t^{2}\right)^{2}}{4}\left\|\nabla u^{+}\right\|_{2}^{2}\left\|\nabla u^{-}\right\|_{2}^{2} \\
& +\frac{\lambda}{4} \int_{\Omega}\left[\left(s^{2}-t^{2}\right) s^{2} \phi_{u^{-}}\left(u^{+}\right)^{2}\right. \\
& \left.+\left(t^{2}-s^{2}\right) t^{2} \phi_{u^{+}}\left(u^{-}\right)^{2}\right] d x, \forall u=u^{+}+u^{-} \in H, s, t \geq 0 .
\end{aligned}
$$

Proof. According to $\left(g_{4}\right)$, we can deduce that

$$
\begin{aligned}
& \frac{1-t^{4}}{4} g(\tau) \tau+G(t \tau)-G(\tau)+\frac{a \theta_{0} \lambda_{1} \tau^{2}\left(1-t^{2}\right)^{2}}{4} \\
& \quad=\int_{t}^{1}\left[\frac{g(\tau)}{\tau^{3}}-\frac{g(s \tau)}{(s \tau)^{3}}+\frac{a \theta_{0} \lambda_{1}\left(1-s^{2}\right)}{(s \tau)^{2}}\right] s^{3} \tau^{4} d s \geq 0, \forall t \geq 0, \tau \in \mathbb{R} \backslash\{0\}
\end{aligned}
$$


From (9), (12), (13), and (21), we have

$$
\begin{aligned}
& F(u)-F\left(s u^{+}+t u^{-}\right)=\frac{a}{2}\left\{\|\nabla u\|_{2}^{2}-\left\|s \nabla u^{+}+t \nabla u^{-}\right\|_{2}^{2}\right\} \\
& +\frac{b}{4}\left\{\|\nabla u\|_{2}^{4}-\left\|s \nabla u^{+}+t \nabla u^{-}\right\|_{2}^{4}\right\} \\
& +\frac{\lambda}{4} \int_{\Omega}\left[\phi_{u} u^{2}-\phi_{s u^{+}+t u^{-}}\left(s u^{+}+t u^{-}\right)^{2}\right] d x \\
& +\int_{\Omega}\left[G\left(s u^{+}+t u^{-}\right)-G(u)\right] d x \\
& =\frac{1-s^{4}}{4}\left\{a\left\|\nabla u^{+}\right\|_{2}^{2}+b\|\nabla u\|_{2}^{2}\left\|\nabla u^{+}\right\|_{2}^{2}\right. \\
& \left.+\int_{\Omega} \lambda \phi_{u}\left(u^{+}\right)^{2} d x-\int_{\Omega} g\left(u^{+}\right) u^{+} d x\right\} \\
& +\frac{1-t^{4}}{4}\left\{a\left\|\nabla u^{-}\right\|_{2}^{2}+b\|\nabla u\|_{2}^{2}\left\|\nabla u^{-}\right\|_{2}^{2}\right. \\
& \left.+\int_{\Omega} \lambda \phi_{u}\left(u^{-}\right)^{2} d x-\int_{\Omega} g\left(u^{-}\right) u^{-} d x\right\} \\
& +\frac{a\left(1-s^{2}\right)^{2}}{4}\left\|\nabla u^{+}\right\|_{2}^{2}+\frac{a\left(1-t^{2}\right)^{2}}{4}\left\|\nabla u^{-}\right\|_{2}^{2} \\
& +\frac{b\left(s^{2}-t^{2}\right)^{2}}{4}\left\|\nabla u^{+}\right\|_{2}^{2}\left\|\nabla u^{-}\right\|_{2}^{2} \\
& +\frac{\lambda}{4} \int_{\Omega}\left[\left(s^{2}-t^{2}\right) s^{2} \phi_{u^{-}}\left(u^{+}\right)^{2}\right. \\
& \left.+\left(t^{2}-s^{2}\right) t^{2} \phi_{u^{+}}\left(u^{-}\right)^{2}\right] d x \\
& +\int_{\Omega}\left[\frac{1-s^{4}}{4} g\left(u^{+}\right) u^{+}-G\left(u^{+}\right)+G\left(s u^{+}\right)\right] d x \\
& +\int_{\Omega}\left[\frac{1-t^{4}}{4} g\left(u^{-}\right) u^{-}-G\left(u^{-}\right)+G\left(t u^{-}\right)\right] d x \\
& \geq \frac{1-s^{4}}{4}\left\langle F^{\prime}(u), u^{+}\right\rangle+\frac{1-t^{4}}{4}\left\langle F^{\prime}(u), u^{-}\right\rangle \\
& +\frac{b\left(s^{2}-t^{2}\right)^{2}}{4}\left\|\nabla u^{+}\right\|_{2}^{2}\left\|\nabla u^{-}\right\|_{2}^{2} \\
& +\frac{a\left(1-s^{2}\right)^{2}\left(1-\theta_{0}\right)}{4}\left\|\nabla u^{+}\right\|_{2}^{2} \\
& +\frac{a\left(1-t^{2}\right)^{2}\left(1-\theta_{0}\right)}{4}\left\|\nabla u^{-}\right\|_{2}^{2} \\
& +\frac{\lambda}{4} \int_{\Omega}\left[\left(s^{2}-t^{2}\right) s^{2} \phi_{u^{-}}\left(u^{+}\right)^{2}\right. \\
& \left.+\left(t^{2}-s^{2}\right) t^{2} \phi_{u^{+}}\left(u^{-}\right)^{2}\right] d x \\
& +\int_{\Omega}\left[\frac{1-s^{4}}{4} g\left(u^{+}\right) u^{+}+G\left(s u^{+}\right)\right. \\
& \left.-G\left(u^{+}\right)+\frac{a\left(1-s^{2}\right)^{2} \theta_{0} \lambda_{1}}{4}\left|u^{+}\right|^{2}\right] d x \\
& +\int_{\Omega}\left[\frac{1-t^{4}}{4} g\left(u^{-}\right) u^{-}+G\left(t u^{-}\right)-G\left(u^{-}\right)\right. \\
& \left.+\frac{a\left(1-t^{2}\right)^{2} \theta_{0} \lambda_{1}}{4}\left|u^{-}\right|^{2}\right] d x \\
& \geq \frac{1-s^{4}}{4}\left\langle F^{\prime}(u), u^{+}\right\rangle+\frac{1-t^{4}}{4}\left\langle F^{\prime}(u), u^{-}\right\rangle \\
& +\frac{b\left(s^{2}-t^{2}\right)^{2}}{4}\left\|\nabla u^{+}\right\|_{2}^{2}\left\|\nabla u^{-}\right\|_{2}^{2} \\
& +\frac{a\left(1-s^{2}\right)^{2}\left(1-\theta_{0}\right)}{4}\left\|\nabla u^{+}\right\|_{2}^{2} \\
& +\frac{a\left(1-t^{2}\right)^{2}\left(1-\theta_{0}\right)}{4}\left\|\nabla u^{-}\right\|_{2}^{2} \\
& +\frac{\lambda}{4} \int_{\Omega}\left[\left(s^{2}-t^{2}\right) s^{2} \phi_{u^{-}}\left(u^{+}\right)^{2}+\left(t^{2}-s^{2}\right) t^{2} \phi_{u^{+}}\left(u^{-}\right)^{2}\right] d x .
\end{aligned}
$$

The above inequality implies that (20) holds.
Corollary 6. If the assumptions $\left(g_{1}\right)-\left(g_{4}\right)$ hold and $u=u^{+}$ $+u^{-} \in \mathscr{M}$, then

$$
\begin{aligned}
F(u) \geq & F\left(s u^{+}+t u^{-}\right)+\frac{a\left(1-s^{2}\right)^{2}\left(1-\theta_{0}\right)}{4}\left\|\nabla u^{+}\right\|_{2}^{2} \\
& +\frac{a\left(1-t^{2}\right)^{2}\left(1-\theta_{0}\right)}{4}\left\|\nabla u^{-}\right\|_{2}^{2} \\
& +\frac{b\left(s^{2}-t^{2}\right)^{2}}{4}\left\|\nabla u^{+}\right\|_{2}^{2}\left\|\nabla u^{-}\right\|_{2}^{2} \\
& +\frac{\lambda}{4} \int_{\Omega}\left[\left(s^{2}-t^{2}\right) s^{2} \phi_{u^{-}}\left(u^{+}\right)^{2}\right. \\
& \left.+\left(t^{2}-s^{2}\right) t^{2} \phi_{u^{+}}\left(u^{-}\right)^{2}\right] d x, \forall s, t \geq 0 .
\end{aligned}
$$

From $u \in \mathscr{M}$, we have $\left\langle F^{\prime}(u), u^{+}\right\rangle=\left\langle F^{\prime}(u), u^{-}\right\rangle=0$. Therefore, we can immediately get the above conclusion by (20).

$$
F\left(u^{+}+u^{-}\right)=\max _{s, t \geq 0} F\left(s u^{+}+t u^{-}\right) .
$$

Lemma 7. Assume that $\left(g_{4}\right)$ holds, then

$$
\frac{1}{4} g(\tau) \tau-G(\tau)+\frac{a \theta_{0} \lambda_{1}}{4} \tau^{2} \geq 0, \forall \tau \in \mathbb{R}
$$

We can get (25) by taking $t=0$ in (21).

Lemma 8. If the assumptions $\left(g_{1}\right)-\left(g_{4}\right)$ hold, then for any $u \in H$, we have

$$
\begin{aligned}
F(u) \geq & F(t u)+\frac{1-t^{4}}{4}\left\langle F^{\prime}(u), u>\right. \\
& +\frac{a\left(1-\theta_{0}\right)\left(1-t^{2}\right)^{2}}{4}\|\nabla u\|_{2}^{2}, \forall t \geq 0 .
\end{aligned}
$$

We can get the conclusion by a similar deduction as Lemma 5

Corollary 9. If the assumptions $\left(g_{1}\right)-\left(g_{4}\right)$ hold and $u \in \mathcal{N}$, then

$$
\begin{aligned}
& F(u) \geq F(t u)+\frac{a\left(1-\theta_{0}\right)\left(1-t^{2}\right)^{2}}{4}\|\nabla u\|_{2}^{2}, \\
& F(u)=\max _{t \geq 0} F(t u), \forall t \geq 0 .
\end{aligned}
$$

\section{Some Critical Preliminaries}

Lemma 10. If the assumptions $\left(g_{1}\right)-\left(g_{4}\right)$ hold and $u \in H$ with $u^{ \pm} \neq 0$, then there exists a unique pair $\left(s_{u}, t_{u}\right)$ of positive numbers such that $s_{u} u^{+}+t_{u} u^{-} \in \mathscr{M}$.

Proof. From the definition of the set $\mathscr{M}, s_{u} u^{+}+t_{u} u^{-} \in \mathscr{M}$ implies that $\left\langle F^{\prime}\left(s_{u} u^{+}+t_{u} u^{-}\right), s_{u} u^{+}>=\left\langle F^{\prime}\left(s_{u} u^{+}+t_{u} u^{-}\right)\right.\right.$, $t_{u} u^{-}>=0$. Thus, we assume 


$$
\begin{aligned}
f_{1}(s, t)=<F^{\prime}\left(s u^{+}+t u^{-}\right), s u^{+}> \\
=a s^{2}\left\|\nabla u^{+}\right\|_{2}^{2}+b s^{4}\left\|\nabla u^{+}\right\|_{2}^{4}+b s^{2} t^{2}\left\|\nabla u^{+}\right\|_{2}^{2}\left\|\nabla u^{-}\right\|_{2}^{2} \\
\quad+s^{4} \int_{\Omega} \lambda \phi_{u^{+}}\left(u^{+}\right)^{2} d x+s^{2} t^{2} \int_{\Omega} \lambda \phi_{u^{-}}\left(u^{+}\right)^{2} d x \\
\quad-\int_{\Omega} g\left(s u^{+}\right) s u^{+} d x,
\end{aligned}
$$

$$
\begin{aligned}
f_{2}(s, t)=<F^{\prime}\left(s u^{+}+t u^{-}\right), t u^{-}> \\
=a t^{2}\left\|\nabla u^{-}\right\|_{2}^{2}+b t^{4}\left\|\nabla u^{-}\right\|_{2}^{4}+b s^{2} t^{2}\left\|\nabla u^{+}\right\|_{2}^{2}\left\|\nabla u^{-}\right\|_{2}^{2} \\
\quad+t^{4} \int_{\Omega} \lambda \phi_{u^{-}}\left(u^{-}\right)^{2} d x+s^{2} t^{2} \int_{\Omega} \lambda \phi_{u^{+}}\left(u^{-}\right)^{2} d x \\
\quad-\int_{\Omega} g\left(t u^{-}\right) t u^{-} d x .
\end{aligned}
$$

If there is a unique pair $(s, t)$ of positive numbers such that $g_{1}(s, t)=g_{2}(s, t)=0$, then Lemma 10 holds. Next, we will give the detailed proof.

By $\left(g_{1}\right)$ and $\left(g_{2}\right)$, we have that $f_{1}(s, s)>0, f_{2}(s, s)>0$ for $s>0$ small enough and $f_{1}(t, t)<0, f_{2}(t, t)<0$ for $t>0$ large enough. Thus, there exists $0<\alpha<\beta$ such that

$$
f_{1}(\alpha, \alpha)>0, f_{2}(\alpha, \alpha)>0, f_{1}(\beta, \beta)<0, f_{2}(\beta, \beta)<0
$$

According to (28) and (29), it is clear that $f_{1}(s, t)$ is increasing on $t$ for fixed $s$ and $f_{2}(s, t)$ is increasing on $s$ for fixed $t$. Thus, combining (30) we have

$$
\begin{aligned}
& f_{1}(\alpha, t)>0, f_{1}(\beta, t)<0, \forall t \in[\alpha, \beta], \\
& f_{2}(s, \alpha)>0, f_{2}(s, \beta)<0, \forall s \in[\alpha, \beta] .
\end{aligned}
$$

Miranda's theorem [20] implies that there exists some point $\left(s_{u}, t_{u}\right)$ such that $f_{1}\left(s_{u}, t_{u}\right)=f_{2}\left(s_{u}, t_{u}\right)=0$, where $s_{u}$, $t_{u} \in(\alpha, \beta)$. Therefore, there exists a positive pair of numbers $\left(s_{u}, t_{u}\right)$ such that $s_{u} u^{+}+t_{u} u^{-} \in \mathscr{M}$.

Next, we will prove that $\left(s_{u}, t_{u}\right)$ is unique for (28) and (29). Let $\left(s_{1}, t_{1}\right)$ and $\left(s_{2}, t_{2}\right)$ be such that $s_{i} u^{+}+t_{i} u^{-} \in \mathscr{M}$. From Corollary 6, we have

$$
\begin{aligned}
& F\left(s_{1} u^{+}+t_{1} u^{-}\right) \geq F\left(\frac{s_{2}}{s_{1}} s_{1} u^{+}+\frac{t_{2}}{t_{1}} t_{1} u^{-}\right), \\
& F\left(s_{2} u^{+}+t_{2} u^{-}\right) \geq F\left(\frac{s_{1}}{s_{2}} s_{2} u^{+}+\frac{t_{1}}{t_{2}} t_{2} u^{-}\right) .
\end{aligned}
$$

The above two inequalities implies that $F\left(s_{1} u^{+}+t_{1} u^{-}\right)$ $=F\left(s_{2} u^{+}+t_{2} u^{-}\right)$, that is, $\left(s_{1}, t_{1}\right)=\left(s_{2}, t_{2}\right)$. The uniqueness is proved. Therefore, Lemma 10 holds.

Corollary 11. If the assumptions $\left(g_{1}\right)-\left(g_{4}\right)$ hold and $u \in$ $H_{0}^{1}(\Omega) \backslash\{0\}$, then there exists a unique $t_{u}>0$ such that $t_{u} u \in \mathcal{N}$.
Lemma 12. If the assumptions $\left(g_{1}\right)-\left(g_{4}\right)$ hold, then

$$
\inf _{u \in \mathscr{M}} F(u)=m=\inf _{u \in H, u^{ \pm} \neq 0} \max _{s, t \geq 0} F\left(s u^{+}+t u^{-}\right) .
$$

Proof. Firstly, by Corollary 6, one has

$$
\inf _{u \in H, u^{ \pm} \neq 0} \max _{s, t \geq 0} F\left(s u^{+}+t u^{-}\right) \leq \inf _{u \in \mathscr{M}} \max _{s, t \geq 0} F\left(s u^{+}+t u^{-}\right)=\inf _{u \in \mathscr{M}} F(u)=m .
$$

Secondly, for any $u \in H$ with $u^{ \pm} \neq 0$, it follows from Lemma 10 that

$$
\max _{s, t \geq 0} F\left(s u^{+}+t u^{-}\right) \geq F\left(s_{u} u^{+}+t_{u} u^{-}\right) \geq \inf _{v \in \mathscr{M}} F(v)=m
$$

Combining (35) and (36), we can get Lemma 12.

Lemma 13. If the assumptions $\left(g_{1}\right)-\left(g_{4}\right)$ hold, then $m>0$ can be achieved.

Proof. For all $u \in \mathscr{M}$, we have $\left\langle F^{\prime}(u), u\right\rangle=0$. According to $\left(g_{1}\right),\left(g_{3}\right)$, and the Sobolev embedding theorem, we can get

$$
\begin{aligned}
a\|\nabla u\|_{2}^{2} & \leq a\|\nabla u\|_{2}^{2}+b\|\nabla u\|_{2}^{4}+\lambda \int_{\Omega} \phi_{u} u^{2} d x \\
& =\int_{\Omega} g(u) u d x \leq \frac{a \lambda_{1}}{2}\|u\|_{2}^{2}+c\|u\|_{5}^{5} \\
& \leq \frac{a}{2}\|\nabla u\|_{2}^{2}+c_{5}\|\nabla u\|_{2}^{5},
\end{aligned}
$$

where $c$ and $c_{5}$ are positive constants. Thus we have $\|\nabla u\|_{2}^{2} \geq\left(a / 2 c_{5}\right)^{2 / 3}>0$. Therefore, by (9), (10), (25), and (37), we have

$$
\begin{aligned}
F(u) & =F(u)-\frac{1}{4}\left\langle F^{\prime}(u), u\right\rangle \\
& =\frac{a}{4}\|\nabla u\|_{2}^{2}+\int_{\Omega} \frac{1}{4} g(u) u-G(u) d x \\
& \geq \frac{a}{4}\|\nabla u\|_{2}^{2}-\frac{a \theta_{0} \lambda_{1}}{4}\|u\|_{2}^{2} \\
& \geq \frac{a\left(1-\theta_{0}\right)}{4}\|\nabla u\|_{2}^{2} \\
& \geq \frac{a\left(1-\theta_{0}\right)}{4}\left(\frac{a}{2 c_{5}}\right)^{2 / 3}>0 .
\end{aligned}
$$

Since $\theta_{0} \in(0,1)$, thus, for any $u \in \mathscr{M}, F(u)>0$ and $m>0$. Let $\left\{u_{n}\right\} \subset \mathscr{M}$ be such that $F\left(u_{n}\right) \longrightarrow m$. For large $n \in \mathbb{N}$, one has

$$
m+1 \geq F\left(u_{n}\right)-\frac{1}{4}<F^{\prime}\left(u_{n}\right), u_{n}>\geq \frac{a\left(1-\theta_{0}\right)}{4}\left\|\nabla u_{n}\right\|_{2}^{2} .
$$


Thus, $\left\{u_{n}\right\}$ is bounded in $H_{0}^{1}(\Omega)$ for $\theta_{0} \in(0,1)$, then there exists $u_{0} \in H$ such that $u_{n}^{ \pm} \rightarrow u_{0}^{ \pm}$in $H$. From $u_{n} \in \mathscr{M}$, we have $\left\langle F^{\prime}\left(u_{n}\right), u_{n}^{ \pm}\right\rangle=0$, that is

$a\left\|\nabla u_{n}^{ \pm}\right\|_{2}^{2}+b\left\|\nabla u_{n}\right\|_{2}^{2}\left\|\nabla u_{n}^{ \pm}\right\|_{2}^{2}+\int_{\Omega} \lambda \phi_{u_{n}}\left(u_{n}^{ \pm}\right)^{2} d x=\int_{\Omega} g\left(u_{n}^{ \pm}\right) u_{n}^{ \pm} d x$.

By a similar deduction as (37), we have $\left\|\nabla u_{n}\right\|_{2}^{2} \geq \mu$ for all $n \in \mathbb{N}$. From $\left(g_{1}\right)$ and $\left(g_{3}\right)$, for any $\varepsilon>0$, there exists $c_{\varepsilon}>0$ such that

$$
g(s) s \leq \varepsilon s^{2}+c_{\varepsilon} s^{5}, \forall s \in \mathbb{R} .
$$

Thus, $\mu \leq\left\|\nabla u_{n}^{ \pm}\right\|_{2}^{2} \leq 1 / a \int_{\Omega} g\left(u_{n}^{ \pm}\right) u_{n}^{ \pm} d x \leq \varepsilon / a\left\|u_{n}^{ \pm}\right\|_{2}^{2}+\left(c_{\varepsilon} / a\right)$ $\left\|u_{n}^{ \pm}\right\|_{5}^{5}$. Since $\left\{u_{n}\right\}$ is bounded in $H$, there is $c_{1}>0$ such that $\int_{\Omega}\left|u_{n}^{ \pm}\right|^{2} d x \leq c_{1}$, which implies

$$
\mu \leq \frac{\varepsilon c_{1}}{a}+\frac{c_{\varepsilon}}{a}\left\|u_{n}^{ \pm}\right\|_{5}^{5} .
$$

Choose $\varepsilon=a \mu / 2 c_{1}$, then $\mu / 2 \leq c_{\varepsilon} / a\left\|u_{n}^{ \pm}\right\|_{5}^{5}$, that is $\left\|u_{n}^{ \pm}\right\|_{5}^{5}$ $\geq a \mu / 2 c_{\varepsilon}$. By the compactness of the embedding $H_{0}^{1}(\Omega)^{\circ}$ $L^{q}(\Omega)$ for $q \in[2,6)$, we have

$$
\int_{\Omega}\left|u_{0}^{ \pm}\right|^{5} d x \geq \frac{a \mu}{2 c_{\varepsilon}} .
$$

Thus $u_{0}^{ \pm} \neq 0$. By $\left(g_{1}\right),\left(g_{3}\right)$, and the compactness lemma of Strauss [21], we have

$$
\begin{gathered}
\lim _{n \rightarrow \infty} \int_{\Omega} g\left(u_{n}^{ \pm}\right) u_{n}^{ \pm} d x=\int_{\Omega} g\left(u_{0}^{ \pm}\right) u_{0}^{ \pm} d x, \\
\lim _{n \rightarrow \infty} \int_{\Omega} G\left(u_{n}^{ \pm}\right) d x=\int_{\Omega} G\left(u_{0}^{ \pm}\right) d x .
\end{gathered}
$$

Also, according to the properties of the solution for the Poisson system, we have

$$
\liminf _{n \rightarrow \infty} \int_{\Omega} \phi_{u_{n}}\left|u_{n}^{ \pm}\right|^{2} d x=\int_{\Omega} \phi_{u_{0}}\left|u_{0}^{ \pm}\right|^{2} d x .
$$

By the weak semicontinuity of norm, we have

$$
\begin{aligned}
& a\left\|\nabla u_{0}^{ \pm}\right\|_{2}^{2}+b\left\|\nabla u_{0}\right\|_{2}^{2}\left\|\nabla u_{0}^{ \pm}\right\|_{2}^{2} \\
& \quad \leq \liminf _{n \rightarrow \infty}\left\{a\left\|\nabla u_{n}^{ \pm}\right\|_{2}^{2}+b\left\|\nabla u_{n}\right\|_{2}^{2}\left\|\nabla u_{n}^{ \pm}\right\|_{2}^{2}\right\} .
\end{aligned}
$$

From (40), (44), (46), and (47), we have

$$
\begin{array}{r}
a\left\|\nabla u_{0}^{ \pm}\right\|_{2}^{2}+b\left\|\nabla u_{0}\right\|_{2}^{2}\left\|\nabla u_{0}^{ \pm}\right\|_{2}^{2}+\int_{\Omega} \phi_{u_{0}}\left|u_{0}^{ \pm}\right|^{2} d x \\
\quad \leq \liminf _{n \rightarrow \infty} \int_{\Omega} g\left(u_{n}^{ \pm}\right) u_{n}^{ \pm} d x=\int_{\Omega} g\left(u_{0}^{ \pm}\right) u_{0}^{ \pm} d x,
\end{array}
$$

that is,

$$
<F^{\prime}\left(u_{0}\right), u_{0}^{ \pm}>\leq 0 \text {. }
$$

Since $\|\nabla u\|_{2}^{2}-\theta_{0} \lambda_{1}\|u\|_{2}^{2} \geq\left(1-\theta_{0}\right)\|\nabla u\|_{2}^{2}$, for all $u \in H$, by (9), (10), (20), and (25), the weak semicontinuity of norm, Fatou's lemma, and Lemma 12, we can get

$$
\begin{aligned}
m= & \lim _{n \rightarrow \infty}\left\{F\left(u_{n}\right)-\frac{1}{4}<F^{\prime}\left(u_{n}\right), u_{n}>\right\} \\
= & \lim _{n \rightarrow \infty}\left\{\frac{a}{4}\left\|\nabla u_{n}\right\|_{2}^{2}+\int_{\Omega} \frac{1}{4}\left[g\left(u_{n}\right) u_{n}-G\left(u_{n}\right)\right] d x\right\} \\
\geq & \frac{a}{4} \liminf _{n \rightarrow \infty}\left(\left\|\nabla u_{n}\right\|_{2}^{2}-\theta_{0} \lambda_{1}\left\|u_{n}\right\|_{2}^{2}\right) \\
& +\liminf _{n \rightarrow \infty} \int_{\Omega}\left(\frac{1}{4} g\left(u_{n}\right) u_{n}-G\left(u_{n}\right)+\frac{a \theta_{0} \lambda_{1}}{4}\left|u_{n}\right|^{2}\right) d x \\
\geq & \frac{a}{4}\left(\left\|\nabla u_{0}\right\|_{2}^{2}-\theta_{0} \lambda_{1}\left\|u_{0}\right\|_{2}^{2}\right) \\
& +\int_{\Omega}\left(\frac{1}{4} g\left(u_{0}\right) u_{0}-G\left(u_{0}\right)+\frac{a \theta_{0} \lambda_{1}}{4}\left|u_{0}\right|^{2}\right) \\
= & \frac{a}{4}\left\|\nabla u_{0}\right\|_{2}^{2}+\int_{\Omega}\left[\frac{1}{4} g\left(u_{0}\right) u_{0}-G\left(u_{0}\right)\right] d x \\
= & F\left(u_{0}\right)-\frac{1}{4}<F^{\prime}\left(u_{0}\right), u_{0}> \\
\geq & \sup _{s, t \geq 0}\left[F\left(s u_{0}^{+}+t u_{0}^{-}\right)+\frac{1-s^{4}}{4}<F^{\prime}\left(u_{0}\right), u_{0}^{+}>\right. \\
& \left.+\frac{1-t^{4}}{4}<F^{\prime}\left(u_{0}\right), u_{0}^{-}>\right]-\frac{1}{4}<F^{\prime}\left(u_{0}\right), u_{0}> \\
= & \sup _{s, t \geq 0}\left[F\left(s u_{0}^{+}+t u_{0}^{-}\right)-\frac{s^{4}}{4}<F^{\prime}\left(u_{0}\right), u_{0}^{+}>\right. \\
& \left.-\frac{t^{4}}{4}<F^{\prime}\left(u_{0}\right), u_{0}^{-}>\right] \\
\geq & \max _{s, t \geq 0} F\left(s u_{0}^{+}+t u_{0}^{-}\right) \geq m . \\
&
\end{aligned}
$$

Thus, $\quad \lim _{n \rightarrow \infty}\left(\left\|\nabla u_{n}\right\|_{2}^{2}-\theta_{0} \lambda_{1}\left\|u_{n}\right\|_{2}^{2}\right)=\left\|\nabla u_{0}\right\|_{2}^{2}-\theta_{0} \lambda_{1}\left\|u_{0}\right\|_{2}^{2}$. Consequently, $u_{n} \longrightarrow u_{0}$ in $H_{0}^{1}(\Omega)$ and $F\left(u_{0}\right)=m, u_{0} \in \mathscr{M}$.

Corollary 14. Assume that $\left(g_{1}\right)-\left(g_{4}\right)$ hold. Then

$$
\inf _{u \in \mathcal{N}} F(u)=c=\inf _{u \in H, u \neq 0} \max _{t \geq 0} F(t u)
$$

and $c>0$.

Lemma 15 (See for example [3]). Assume that $\left(g_{1}\right)-\left(g_{4}\right)$ hold. Then there exists a constant $c_{*} \in(0, c]$ and a sequence $\left\{u_{n}\right\} \subset$ E satisfying

$$
F\left(u_{n}\right) \longrightarrow c_{*},\left\|F^{\prime}\left(u_{n}\right)\right\|\left(1+\left\|u_{n}\right\|\right) \longrightarrow 0 .
$$


Lemma 16. If the assumptions $\left(g_{1}\right)-\left(g_{4}\right)$ hold and $u_{0} \in \mathscr{M}$ with $F\left(u_{0}\right)=m$, then $u_{0}$ is a critical point of $F$.

Proof. For $F^{\prime}\left(u_{0}\right) \neq 0$, there exist $\sigma>0$ and $\rho>0$ such that

$$
u \in H_{0}^{1}(\Omega),\left\|u-u_{0}\right\| \leq 3 \sigma \Longrightarrow\left\|F^{\prime}(u)\right\| \geq \rho .
$$

From (23), we have that

$$
\begin{aligned}
F\left(s u_{0}^{+}+t u_{0}^{-}\right) \leq & F\left(u_{0}\right)-\frac{a\left(1-\theta_{0}\right)\left(1-s^{2}\right)^{2}}{4}\left\|\nabla u_{0}^{+}\right\|_{2}^{2} \\
& -\frac{a\left(1-\theta_{0}\right)\left(1-t^{2}\right)^{2}}{4}\left\|\nabla u_{0}^{-}\right\|_{2}^{2} \\
= & m-\frac{a\left(1-\theta_{0}\right)\left(1-s^{2}\right)^{2}}{4}\left\|\nabla u_{0}^{+}\right\|_{2}^{2} \\
& -\frac{a\left(1-\theta_{0}\right)\left(1-t^{2}\right)^{2}}{4}\left\|\nabla u_{0}^{-}\right\|_{2}^{2} \forall s, t \geq 0 .
\end{aligned}
$$

Let $D=(1 / 2,3 / 2) \times(1 / 2,3 / 2), g(s, t)=s u_{0}^{+}+t u_{0}^{-}$. It follows from (54) that

$$
\chi=\max _{(s, t) \in \partial D} I\left(s u_{0}^{+}+t u_{0}^{-}\right)<m .
$$

For $\varepsilon=\min \{(m-\chi) / 3,1, \rho \sigma / 8\}, S=B\left(u_{0}, \sigma\right), \quad$ [22]. Lemma 7 yields a deformation $\eta \in C([0,1] \times H, H)$ such that

(i) $\eta(1, u)=u$, if $u \notin F^{-1}([m-2 \varepsilon, m+2 \varepsilon]) \cap S_{2 \sigma}$

(ii) $\eta\left(1, F^{m+\varepsilon} \cap B\left(u_{0}, \sigma\right)\right) \subset F^{m-\varepsilon}$

(iii) $F(\eta(1, u)) \leq F(u), \forall u \in H_{0}^{1}(\Omega)$.

From Corollary 6, we have $F\left(s u_{0}^{+}+t u_{0}^{-}\right) \leq F\left(u_{0}\right)=m$ for $s, t \geq 0$. For $s, t \geq 0,|s-1|^{2}+|t-1|^{2}<\sigma^{2} /\left\|u_{0}\right\|^{2}$, we know $s$ $u_{0}^{+}+t u_{0}^{-} \in F^{m+\varepsilon} \cap B\left(u_{0}, \sigma\right)$, then it follows from (ii) that $F(s$ $\left.u_{0}^{+}+t u_{0}^{-}\right) \leq m-\varepsilon$.

According to (iii) and (54), we have that

$$
\begin{aligned}
F\left(\eta\left(1, s u_{0}^{+}+t u_{0}^{-}\right)\right) & \leq \\
\leq & \left(s u_{0}^{+}+t u_{0}^{-}\right) \\
\leq & m-\frac{a\left(1-\theta_{0}\right)\left(1-s^{2}\right)^{2}}{4}\left\|\nabla u_{0}^{+}\right\|_{2}^{2} \\
& -\frac{a\left(1-\theta_{0}\right)\left(1-t^{2}\right)^{2}}{4}\left\|\nabla u_{0}^{-}\right\|_{2}^{2} \\
\leq & m-\frac{a\left(1-\theta_{0}\right)}{4} \min \left\{\left\|\nabla u_{0}^{+}\right\|_{2}^{2},\left\|\nabla u_{0}^{-}\right\|_{2}^{2}\right\} \\
& \cdot\left[\left(1-s^{2}\right)^{2}+\left(1-t^{2}\right)^{2}\right] \\
\leq & m-\frac{a\left(1-\theta_{0}\right) \sigma^{2}}{4\left\|u_{0}\right\|^{2}} \min \left\{\left\|\nabla u_{0}^{+}\right\|_{2}^{2},\left\|\nabla u_{0}^{-}\right\|_{2}^{2}\right\}, \\
\forall s, t \geq & 0,|s-1|^{2}+|t-1|^{2} \geq \sigma^{2} /\left\|u_{0}\right\|^{2} .
\end{aligned}
$$

Thus, $\max _{s, t \in \bar{D}} F(\eta(1, g(s, t)))<m$.

Next, we prove that $\eta(1, g(D)) \bigcap \mathscr{M} \neq \varnothing$, which contradicts to the definition of $m$. Let us define $h(s, t)=\eta(1, g$ $(s, t))$ and

$$
\begin{aligned}
\Psi_{0}(s, t) & =\left(F^{\prime}(g(s, t)) u_{0}^{+}, F^{\prime}(g(s, t)) u_{0}^{-}\right) \\
& =\left(F^{\prime}\left(s u_{0}^{+}+t u_{0}^{-}\right) u_{0}^{+}, F^{\prime}\left(s u_{0}^{+}+t u_{0}^{-}\right) u_{0}^{-}\right), \\
\Psi_{1}(s, t) & =\left(\frac{1}{s} F^{\prime}(h(s, t)) h^{+}(s, t), \frac{1}{t} F^{\prime}(h(s, t)) h^{-}(s, t)\right) .
\end{aligned}
$$

Lemma 10 and the degree theory yields $\operatorname{deg}\left(\Psi_{0}(s, t)\right.$, $D, 0)=1$. By (55), we can deduce that $g=h$ on $\partial \Omega$. Consequently, $\operatorname{deg}\left(\Psi_{1}, D, 0\right)=\operatorname{deg}\left(\Psi_{0}, D, 0\right)=1$. Therefore, we have $\Psi_{1}\left(s_{0}, t_{0}\right)=0$ for some $\left(s_{0}, t_{0}\right) \in D$, so that $\eta\left(1, g\left(s_{0}\right.\right.$, $\left.\left.t_{0}\right)\right)=h\left(s_{0}, t_{0}\right) \in \mathscr{M}$, which is a contradiction. Thus, (53) does not hold. In other words, $u_{0}$ is a critical point of $F$, that is, $u_{0}$ is a sign-changing solution for problem (1).

\section{The Existence Result of the Sign- Changing Solutions}

In this section, we mainly give the proof of Theorem 17.

Proof of Theorem 17. By Lemma 13 and Lemma 16, there is a $u_{0} \in \mathscr{M}$ such that $F\left(u_{0}\right)=m$ and $F^{\prime}\left(u_{0}\right)=0$. Therefore, $u_{0}$ is exactly a sign-changing solution of problem (1). Now, we prove that $u_{0}$ changes sign only once.

We assume by contradiction that $u_{0}=u_{1}+u_{2}+u_{3}$, where $u_{i} \neq 0, u_{1} \geq 0, u_{2} \leq 0$ and $\sup p\left(u_{i}\right) \bigcap \sup p\left(u_{j}\right)=\varnothing, i \neq j(i, j$ $=1,2,3$.

Let $v=u_{1}+u_{2}$, then $v^{+}=u_{1}, v^{-}=u_{2}$, and $v^{ \pm} \neq 0$. Note that $\left\langle F^{\prime}\left(u_{0}\right), v^{+}\right\rangle=0$ and $\left\langle F^{\prime}\left(u_{0}\right), v^{-}\right\rangle=0$, we have

$$
\begin{aligned}
& <F^{\prime}(v), v^{+}>=-b\left\|\nabla u_{3}\right\|_{2}^{2}\left\|\nabla v^{+}\right\|_{2}^{2}-\lambda \int_{\Omega} \phi_{u_{3}}\left(v^{+}\right)^{2} d x, \\
& <F^{\prime}(v), v^{-}>=-b\left\|\nabla u_{3}\right\|_{2}^{2}\left\|\nabla v^{-}\right\|_{2}^{2}-\lambda \int_{\Omega} \phi_{u_{3}}\left(v^{-}\right)^{2} d x .
\end{aligned}
$$

From (9)-(13), (23), (25), (58), and (59), we have

$$
\begin{aligned}
m= & F\left(u_{0}\right)-\frac{1}{4}<F^{\prime}\left(u_{0}\right), u_{0}> \\
= & F(v)+F\left(u_{3}\right)+\frac{b}{2}\|\nabla v\|_{2}^{2}\left\|\nabla u_{3}\right\|_{2}^{2} \\
& +\frac{\lambda}{4} \int_{\Omega}\left[\phi_{v}\left(u_{3}\right)^{2}+\phi_{u_{3}}(v)^{2}\right] d x \\
& -\frac{1}{4}\left[<F^{\prime}(v), v>+<F^{\prime}\left(u_{3}\right), u_{3}>+2 b\|\nabla v\|_{2}^{2}\left\|\nabla u_{3}\right\|_{2}^{2}\right. \\
& \left.+\lambda \int_{\Omega}\left[\phi_{v}\left(u_{3}\right)^{2}+\phi_{u_{3}}(v)^{2}\right] d x\right] \\
= & F(v)+F\left(u_{3}\right)-\frac{1}{4}<F^{\prime}(v), v>-\frac{1}{4}<F^{\prime}\left(u_{3}\right), u_{3}> \\
\geq & \sup _{s, t \geq 0}\left\{F\left(s v^{+}+t v^{-}\right)+\frac{1-s^{4}}{4}<F^{\prime}(v), v^{+}>\right. \\
& \left.+\frac{1-t^{4}}{4}<F^{\prime}(v), v^{-}>\right\}+F\left(u_{3}\right)-\frac{1}{4}<F^{\prime}(v), v>
\end{aligned}
$$




$$
\begin{aligned}
&-\frac{1}{4}<F^{\prime}\left(u_{3}\right), u_{3}> \\
& \geq \sup _{s, t \geq 0}\left\{F\left(s v^{+}+t v^{-}\right)+\frac{s^{4}}{4}\left(b\left\|\nabla u_{3}\right\|_{2}^{2}\left\|\nabla v^{+}\right\|_{2}^{2}+\lambda \int_{\Omega} \phi_{u_{3}}\left(v^{+}\right)^{2} d x\right)\right. \\
&\left.+\frac{t^{4}}{4}\left(b\left\|\nabla u_{3}\right\|_{2}^{2}\left\|\nabla v^{-}\right\|_{2}^{2}+\lambda \int_{\Omega} \phi_{u_{3}}\left(v^{-}\right)^{2} d x\right)\right\} \\
&+\frac{a}{4}\left\|\nabla u_{3}\right\|_{2}^{2}+\int_{\Omega}\left[\frac{1}{4} g\left(u_{3}\right) u_{3}-G\left(u_{3}\right)\right] d x \\
& \geq \max _{s, t \geq 0} F\left(s v^{+}+t v^{-}\right)+\frac{a\left(1-\theta_{0}\right)}{4}\left\|\nabla u_{3}\right\|_{2}^{2} \\
& \geq m+\frac{a\left(1-\theta_{0}\right)}{4}\left\|\nabla u_{3}\right\|_{2}^{2} .
\end{aligned}
$$

Since $\theta_{0} \in(0,1)$, we have $u_{3}=0$. Therefore, $u_{0}$ changes sign only once.

\section{Energy Doubling Property}

Under the above preparations, we give the proof of Theorem 18.

Proof of Theorem 18. By Lemma 15, we know that there exists a sequence $\left\{u_{n}\right\} \subset E$ satisfying (52), that is,

$$
F\left(u_{n}\right) \longrightarrow c_{*},<F^{\prime}\left(u_{n}\right), u_{n}>=0 .
$$

According to (9), (10), (25), and (61), we have for large $n \in \mathbb{N}$

$$
c_{*}+1 \geq F\left(u_{n}\right)-\frac{1}{4}<F^{\prime}\left(u_{n}\right), u_{n}>\geq \frac{a\left(1-\theta_{0}\right)}{4}\left\|\nabla u_{n}\right\|_{2}^{2},
$$

which shows that $\left\{u_{n}\right\}$ is bounded in $H_{0}^{1}(\Omega)$ for $\theta_{0} \in(0,1)$. By a standard argument, we can prove that there exists a $u_{1}$ $\in H_{0}^{1}(\Omega) \backslash\{0\}$ such that $F^{\prime}\left(u_{1}\right)=0$. This suggests that $u_{1} \in$ $\mathcal{N}$ is a nontrivial solution for problem (1) and $F\left(u_{1}\right) \geq c$. On the other hand, by using (9), (10), and (25), the weak semicontinuity of norm and Fatou's lemma, we have

$$
\begin{aligned}
c \geq c_{*}= & \lim _{n \rightarrow \infty}\left(F\left(u_{n}\right)-\frac{1}{4}<F^{\prime}\left(u_{n}\right), u_{n}>\right) \\
= & \lim _{n \rightarrow \infty}\left\{\frac{a}{4}\left\|\nabla u_{n}\right\|_{2}^{2}+\int_{\Omega}\left[\frac{1}{4} g\left(u_{n}\right) u_{n}-G\left(u_{n}\right)\right] d x\right\} \\
\geq & \frac{a}{4} \liminf _{n \rightarrow \infty}\left(\left\|\nabla u_{n}\right\|_{2}^{2}+\theta_{0} \lambda_{1}\left\|u_{n}\right\|_{2}^{2}\right) \\
& +\liminf _{n \rightarrow \infty}\left(\int_{\Omega}\left[\frac{1}{4} g\left(u_{n}\right) u_{n}-G\left(u_{n}\right)+\frac{a \theta_{0} \lambda_{1}}{4}\left|u_{n}\right|^{2}\right] d x\right) \\
\geq & \frac{a}{4}\left(\left\|\nabla u_{1}\right\|_{2}^{2}+\theta_{0} \lambda_{1}\left\|u_{1}\right\|_{2}^{2}\right) \\
& +\int_{\Omega}\left[\frac{1}{4} g\left(u_{1}\right) u_{1}-G\left(u_{1}\right)+\frac{a \theta_{0} \lambda_{1}}{4}\left|u_{1}\right|^{2}\right] d x \\
= & \frac{a}{4}\left\|\nabla u_{1}\right\|_{2}^{2}+\int_{\Omega}\left[\frac{1}{4} g\left(u_{1}\right) u_{1}-G\left(u_{1}\right)\right] d x \\
= & F\left(u_{1}\right)-\frac{1}{4}<F^{\prime}\left(u_{1}\right), u_{1}>=F\left(u_{1}\right),
\end{aligned}
$$

which implies $F\left(u_{1}\right) \leq c$. Thus, $F\left(u_{1}\right)=c=\inf _{u \in \mathscr{N}} I>0$. In view of Theorem 1 , there exists $u_{0} \in \mathscr{M}$ such that $I\left(u_{0}\right)=m$. Therefore, from (11), (24), and Corollary 14, we have

$$
\begin{aligned}
& m= F\left(u_{0}\right)=\sup _{s, t \geq 0} F\left(s u_{0}^{+}+t u_{0}^{-}\right) \\
&=\sup _{s, t \geq 0}\left[F\left(s u_{0}^{+}\right)+F\left(t u_{0}^{-}\right)\right. \\
&+\frac{b s^{2} t^{2}}{2}\left\|\nabla u_{0}^{+}\right\|_{2}^{2}\left\|\nabla u_{0}^{-}\right\|_{2}^{2} \\
&\left.\quad+\frac{\lambda s^{2} t^{2}}{4} \int_{\Omega} \phi_{u_{0}^{+}}\left(u_{0}^{-}\right)^{2}+\phi_{u_{0}^{-}}\left(u_{0^{+}}\right)^{2}\right] \\
&>\sup _{s \geq 0} F\left(s u_{0}^{+}\right)+\sup _{t \geq 0} F\left(t u_{0}^{-}\right) \geq 2 c,
\end{aligned}
$$

which implies the energy of the least-energy sign-changing solution is strictly larger than twice that of the ground state solutions of the Nehari type.

\section{The Convergence Property}

In this part, we give the convergence property for $b \searrow 0$. Firstly, we have to give some estimates which will be used in the following process. Choose $\omega_{0} \in C_{0}^{\infty}(\Omega)$ such that $\omega_{0}^{ \pm} \neq 0$, then there exists a constant $c^{\prime}$ such that

$$
\begin{aligned}
\int_{\Omega} & \phi_{s \omega_{0}^{+}+t \omega_{0}^{+}}\left(s \omega_{0}^{+}+t \omega_{0}^{-}\right)^{2} d x \\
& \leq c^{\prime}\left\|s \nabla \omega_{0}^{+}+t \nabla \omega_{0}^{-}\right\|_{2}^{4} \\
& \leq 2 c^{\prime}\left(s^{4}\left\|\nabla \omega_{0}^{+}\right\|_{2}^{4}+t^{4}\left\|\nabla \omega_{0}^{-}\right\|_{2}^{4}\right) .
\end{aligned}
$$

Thus, by $\left(g_{1}\right)$ and $\left(g_{3}\right)$, there exist $\beta_{1}>0$ and $\beta_{2} \geq$ $\max \left\{\left(1+\lambda c^{\prime}\right)\left\|\nabla \omega_{0}^{+}\right\|_{2}^{4} /\left\|\omega_{0}^{+}\right\|_{4}^{4},\left(1+\lambda c^{\prime}\right)\left\|\nabla \omega_{0}^{-}\right\|_{2}^{4} /\left\|\omega_{0}^{-}\right\|_{4}^{4}\right\}$ such that

$$
G(t) \geq \beta_{2}|t|^{4}-\beta_{1}, \forall t \in \mathbb{R}
$$

Proof of Theorem 19. It is clear that $b=0$ is allowed in Section 3. Therefore, we can deduce that there exists a $u_{b_{0}} \in \mathscr{M}_{b_{0}}$ such that $F_{b_{0}}^{\prime}\left(u_{b_{0}}\right)=0$ and $F_{b_{0}}\left(u_{b_{0}}\right)=m_{b_{0}}=$ $\inf _{u \in \mathscr{M}_{b_{0}}} F_{b_{0}}(u)$, that is, problem (2) has the least-energy sign-changing solution, which changes sign only once.

For $b>0$, let $u_{b} \in \mathscr{M}_{b}=\mathscr{M}$ be a sign-changing solution of (1) obtained in Theorem 1, which changes sign only once and satisfies $F_{b}\left(u_{b}\right)=F\left(u_{b}\right)=m_{b}=\inf _{u \in \mathscr{M}_{b}} F_{b}(u)$. 
Then, for any $b \in[0,1]$, it follows from Lemma 12 and (65)-(66), we have

$$
\begin{aligned}
& F_{b}\left(u_{b}\right)=m_{b} \leq \max _{s, t \geq 0} F_{b}\left(s \omega_{0}^{+}+t \omega_{0}^{-}\right) \\
& =\max _{s, t \geq 0}\left(\frac{a s^{2}}{2}\left\|\nabla \omega_{0}^{+}\right\|_{2}^{2}+\frac{b s^{4}}{4}\left\|\nabla \omega_{0}^{+}\right\|_{2}^{4}\right. \\
& -\int_{\Omega} G\left(s \omega_{0}^{+}\right) d x+\frac{a t^{2}}{2}\left\|\nabla \omega_{0}^{-}\right\|_{2}^{2} \\
& +\frac{b t^{4}}{4}\left\|\nabla \omega_{0}^{-}\right\|_{2}^{4}-\int_{\Omega} G\left(t \omega_{0}^{-}\right) d x \\
& +\frac{b s^{2} t^{2}}{2}\left\|\nabla \omega_{0}^{+}\right\|_{2}^{2}\left\|\nabla \omega_{0}^{-}\right\|_{2}^{2} \\
& \left.+\frac{\lambda}{4} \int_{\Omega} \phi_{s \omega_{0}^{+}+t \omega_{0}^{-}}\left(s \omega_{0}^{+}+t \omega_{0}^{-}\right)^{2} d x\right) \\
& \leq \max _{s, t \geq 0}\left(\frac{a s^{2}}{2}\left\|\nabla \omega_{0}^{+}\right\|_{2}^{2}+\frac{b s^{4}}{4}\left\|\nabla \omega_{0}^{+}\right\|_{2}^{4}\right. \\
& -\int_{\Omega} G\left(s \omega_{0}^{+}\right) d x+\frac{a t^{2}}{2}\left\|\nabla \omega_{0}^{-}\right\|_{2}^{2} \\
& +\frac{b t^{4}}{4}\left\|\nabla \omega_{0}^{-}\right\|_{2}^{4}-\int_{\Omega} G\left(t \omega_{0}^{-}\right) d x \\
& +\frac{b s^{4}}{4}\left\|\nabla \omega_{0}^{+}\right\|_{2}^{4}+\frac{b t^{4}}{4}\left\|\nabla \omega_{0}^{-}\right\|_{2}^{4} \\
& +\frac{\lambda c^{\prime}}{2}\left(s^{4}\left\|\nabla \omega_{0}^{+}\right\|_{2}^{4}+t^{4}\left\|\nabla \omega_{0}^{-}\right\|_{2}^{4}\right) \\
& \leq \max _{s, t \geq 0}\left(\frac{a s^{2}}{2}\left\|\nabla \omega_{0}^{+}\right\|_{2}^{2}+\frac{1+\lambda c^{\prime}}{2} s^{4}\left\|\nabla \omega_{0}^{+}\right\|_{2}^{4}\right. \\
& -\int_{\Omega} \beta_{2}\left(s \omega_{0}^{+}\right)^{4} d x+\frac{a t^{2}}{2}\left\|\nabla \omega_{0}^{-}\right\|_{2}^{2} \\
& \left.+\frac{1+\lambda c^{\prime}}{2} t^{4}\left\|\nabla \omega_{0}^{-}\right\|_{2}^{4}-\int_{\Omega} \beta_{2}\left(t \omega_{0}^{-}\right)^{4} d x+2 \beta_{1}|\Omega|\right) \\
& \leq \max _{s, t \geq 0}\left(\frac{a s^{2}}{2}\left\|\nabla \omega_{0}^{+}\right\|_{2}^{2}-\frac{1+\lambda c^{\prime}}{2} s^{4}\left\|\nabla \omega_{0}^{+}\right\|_{2}^{4}\right. \\
& \left.+\frac{a t^{2}}{2}\left\|\nabla \omega_{0}^{-}\right\|_{2}^{2}-\frac{1+\lambda c^{\prime}}{2} t^{4}\left\|\nabla \omega_{0}^{-}\right\|_{2}^{4}\right)+2 \beta_{1}|\Omega| \\
& =\Lambda_{0} \in(0, \infty) \text {. }
\end{aligned}
$$

For any sequence $\left\{b_{n}\right\}$ with $b_{n} \searrow 0$ as $n \longrightarrow \infty$, by (9), (10), (25), and (67), we have for large $n \in \mathbb{N}$

$$
\Lambda_{0}+1 \geq F_{b_{n}}\left(u_{b_{n}}\right)-\frac{1}{4}<F^{\prime} b_{n}\left(u_{b_{n}}\right), u_{b_{n}} \geq \frac{a\left(1-\theta_{0}\right)}{4}\left\|\nabla u_{b_{n}}\right\|_{2}^{2} .
$$

Since $\theta_{0} \in(0,1),\left\{u_{b_{n}}\right\}$ is bounded in $H_{0}^{1}(\Omega)$. Therefore, there exists a subsequence of $\left\{b_{n}\right\}$, still denoted by $\left\{b_{n}\right\}$ and $u_{b_{0}} \in H$, such that $u_{b_{n}} \longrightarrow u_{b_{0}}$ in $H_{0}^{1}(\Omega)$. By a standard argument, we can prove $u_{b_{n}}^{ \pm} \longrightarrow u_{b_{0}}^{ \pm} \neq 0$ in $H_{0}^{1}(\Omega)$. Since

$$
\begin{aligned}
<F_{b_{0}}^{\prime}\left(u_{b_{0}}\right), \varphi>= & a \int_{\Omega} \nabla u_{b_{0}} \nabla \varphi d x+\int_{\Omega} \lambda \phi_{u_{b_{0}}} u_{b_{0}} \varphi d x \\
& -\int_{\Omega} g\left(u_{b_{0}}\right) \varphi d x \\
= & \lim _{n \rightarrow \infty}\left[\left(a+b_{n}\left\|\nabla u_{b_{n}}\right\|_{2}^{2}\right) \int_{\Omega} \nabla u_{b_{n}} \nabla \varphi d x\right. \\
& \left.+\int_{\Omega} \lambda \phi_{u_{b_{n}}} u_{b_{n}} \varphi d x-\int_{\Omega} g\left(u_{b_{n}}\right) \varphi d x\right] \\
= & \lim _{n \rightarrow \infty}<F^{\prime}{ }_{b_{n}}\left(u_{b_{n}}\right), \varphi>=0 . \forall \varphi \in C_{0}^{\infty}(\Omega) .
\end{aligned}
$$

Thus, $F_{b_{0}}^{\prime}\left(u_{b_{0}}\right)=0, u_{b_{0}} \in \mathscr{M}_{b_{0}}$, and $F_{b_{0}}\left(u_{b_{0}}\right) \geq m_{b_{0}}$. Next, we give the proof of $F_{b_{0}}\left(u_{b_{0}}\right)=m_{b_{0}}$. Choose $b_{n} \in[0,1]$, from $\left(g_{2}\right)$, there exists a $K_{0}$ such that

$$
\begin{aligned}
F_{b_{n}}\left(s v_{0}^{+}+t v_{0}^{-}\right)= & \frac{a s^{2}}{2}\left\|\nabla v_{0}^{+}\right\|_{2}^{2}+\frac{b_{n} s^{4}}{4}\left\|\nabla v_{0}^{+}\right\|_{2}^{4}-\int_{\Omega} G\left(s v_{0}^{+}\right) d x \\
& +\frac{a t^{2}}{2}\left\|\nabla v_{0}^{-}\right\|_{2}^{2}+\frac{b_{n} t^{4}}{4}\left\|\nabla v_{0}^{-}\right\|_{2}^{4} \\
& -\int_{\Omega} G\left(t v_{0}^{-}\right) d x+\frac{b_{n} s^{2} t^{2}}{2}\left\|\nabla v_{0}^{+}\right\|_{2}^{2}\left\|\nabla v_{0}^{-}\right\|_{2}^{2} \\
& +\frac{\lambda}{4} \int_{\Omega} \phi_{s v_{0}^{+}+t v_{0}^{-}}\left(s v_{0}^{+}+t v_{0}^{-}\right)^{2} d x \\
\leq & \frac{a s^{2}}{2}\left\|\nabla v_{0}^{+}\right\|_{2}^{2}+\frac{1+\lambda c^{\prime}}{2} s^{4}\left\|\nabla v_{0}^{+}\right\|_{2}^{4} \\
& -\int_{\Omega} G\left(s v_{0}^{+}\right) d x+\frac{a t^{2}}{2}\left\|\nabla v_{0}^{-}\right\|_{2}^{2} \\
& +\frac{1+\lambda c^{\prime}}{2} t^{4}\left\|\nabla v_{0}^{-}\right\|_{2}^{4}-\int_{\Omega} G\left(t v_{0}^{-}\right) d x \\
< & 0, \forall s, t \geq K_{0} .
\end{aligned}
$$

From Lemma 10, there exists $\left(s_{n}, t_{n}\right)$ such that $s_{n} v_{0}^{+}+t_{n}$ $v_{0}^{+} \in \mathscr{M}_{b_{n}}$, (5.4) implies $0<s_{n}, t_{n}<K_{0}$. Since $F_{b_{0}}^{\prime}\left(v_{0}\right)=0$, then from (9), (10), (15), (16), and (20), we have

$$
\begin{aligned}
m_{b_{0}}= & F_{b_{0}}\left(v_{0}\right)=F_{b_{n}}\left(v_{0}\right)-\frac{b_{n}}{4}\left\|\nabla v_{0}\right\|_{2}^{4} \\
\geq & F_{b_{n}}\left(s_{n} v_{0}^{+}+t_{n} v_{0}^{-}\right)+\frac{1-s_{n}^{4}}{4}<F^{\prime} b_{n}\left(v_{0}\right), v_{0}^{+}> \\
& +\frac{1-t_{n}^{4}}{4}<F^{\prime}{ }_{b_{n}}\left(v_{0}\right), v_{0}^{-}>-\frac{b_{n}}{4}\left\|\nabla v_{0}\right\|_{2}^{4} \\
\geq & m_{b_{n}}-\frac{1+K_{0}^{4}}{4}\left|<F^{\prime}{ }_{b_{n}}\left(v_{0}\right), v_{0}^{+}>\right| \\
& -\frac{1+K_{0}^{4}}{4}\left|<F^{\prime}{ }_{b_{n}}\left(v_{0}\right), v_{0}^{-}>\right|-\frac{b_{n}}{4}\left\|\nabla v_{0}\right\|_{2}^{4} \\
= & m_{b_{n}}-\frac{1+K_{0}^{4}}{4} b_{n}\left\|\nabla v_{0}\right\|_{2}^{2}\left\|\nabla v_{0}^{+}\right\|_{2}^{2} \\
& -\frac{1+K_{0}^{4}}{4} b_{n}\left\|\nabla v_{0}\right\|_{2}^{2}\left\|\nabla v_{0}^{-}\right\|_{2}^{2}-\frac{b_{n}}{4}\left\|\nabla v_{0}\right\|_{2}^{4},
\end{aligned}
$$


which implies

$$
\limsup _{n \rightarrow \infty} m_{b_{n}} \leq m_{b_{0}}
$$

According to (9), (15), and (72), we have

$$
m_{b_{0}} \leq F_{b_{0}}\left(u_{b_{0}}\right)=\underset{n \rightarrow \infty}{\limsup _{n}} F_{b_{n}}\left(u_{b_{n}}\right)=\underset{n \rightarrow \infty}{\limsup } m_{b_{n}} \leq m_{b_{0}} .
$$

This shows $F_{b_{0}}\left(u_{b_{0}}\right)=m_{b_{0}}$, and the convergence property of $b \searrow 0$ is proved.

Proof of Theorem 20. Since the proof is similar as the proof of Theorem 19, we omit the details.

\section{Data Availability}

The data used to support the findings of this study are included within the article.

\section{Conflicts of Interest}

The authors declares that they have no conflicts of interest.

\section{Acknowledgments}

The authors would like to express their sincere gratitude to the anonymous referees for their invaluable comments and suggestions which helped improve the paper greatly. This research was supported by the Program for the Innovative Talents of Higher Education Institutions of Shanxi, the Scientific and Technological Innovation Programs of Higher Education Institutions in Shanxi (201802085), and the innovative research team of North University of China(TD201901), the Fund for Shanxi "1331KIRT."

\section{References}

[1] C. O. Alves and M. A. S. Souto, "Existence of least energy nodal solution for a Schrödinger-Poisson system in bounded domains," Zeitschrift Für Angewandte Mathematik und Physik, vol. 65, no. 6, pp. 1153-1166, 2014.

[2] W. Shuai, "Sign-changing solutions for a class of Kirchhofftype problem in bounded domains," Journal of Differential Equations, vol. 259, no. 4, pp. 1256-1274, 2015.

[3] X. H. Tang and B. Cheng, "Ground state sign-changing solutions for Kirchhoff type problems in bounded domains," Journal of Differential Equations, vol. 261, no. 4, pp. 23842402, 2016.

[4] L. Li and J. J. Sun, "Existence and multiplicity of solutions for the Kirchhoff equations with asymptotically linear nonlinearities," Nonlinear Analysis: Real World Applications, vol. 26, pp. 391-399, 2015.

[5] T.-S. Hsu, "Multiple positive solutions for a quasilinear elliptic problem involving critical Sobolev-Hardy exponents and concave-convex nonlinearities," Nonlinear Analysis: Theory, Methods \& Applications, vol. 74, no. 12, pp. 3934-3944, 2011.

[6] J. Guo, S. Ma, and G. Zhang, "Solutions of the autonomous Kirchhoff type equations in RN," Applied Mathematics Letters, vol. 82, pp. 14-17, 2018.
[7] J. Zhang, "Standing waves with a critical frequency for nonlinear Schrödinger equations involving critical growth," Applied Mathematics Letters, vol. 63, pp. 53-58, 2017.

[8] B. Cheng, "Least energy sign-changing solutions for a class of nonlocal Kirchhoff-type problems," Springerplus, vol. 5, no. 1, p. 1256, 2016.

[9] X.-J. Zhong and C.-L. Tang, "Ground state sign-changing solutions for a Schrödinger-Poisson system with a critical nonlinearity in R3," Nonlinear Analysis: Real World Applications, vol. 39, pp. 166-184, 2018.

[10] F. Li, C. Gao, and X. Zhu, "Existence and concentration of sign-changing solutions to Kirchhoff-type system with Hartree-type nonlinearity," Journal of Mathematical Analysis and Applications, vol. 448, no. 1, pp. 60-80, 2017.

[11] S. H. Rasouli, H. Fani, and S. Khademloo, "Existence of signchanging solutions for a nonlocal problem of $\mathrm{p}$-Kirchhoff type," Mediterranean Journal of Mathematics, vol. 14, no. 5, 2017.

[12] Z. Zhang and K. Perera, "Sign changing solutions of Kirchhoff type problems via invariant sets of descent flow," Journal of Mathematical Analysis and Applications, vol. 317, no. 2, pp. 456-463, 2006.

[13] A. Mao, L. Yang, A. Qian, and S. Luan, "Existence and concentration of solutions of Schrödinger-Poisson system," Applied Mathematics Letters, vol. 68, pp. 8-12, 2017.

[14] J. Zhang, Z. Lou, Y. Ji, and W. Shao, "Ground state of Kirchhoff type fractional Schrödinger equations with critical growth," Journal of Mathematical Analysis and Applications, vol. 462, no. 1, pp. 57-83, 2018.

[15] Y. Sun, L. Liu, and Y. Wu, "The existence and uniqueness of positive monotone solutions for a class of nonlinear Schrödinger equations on infinite domains," Journal of Computational and Applied Mathematics, vol. 321, pp. 478-486, 2017.

[16] J. Liu and A. Qian, "Ground state solution for a SchrödingerPoisson equation with critical growth," Nonlinear Analysis: Real World Applications, vol. 40, pp. 428-443, 2018.

[17] T. D'Aprile and D. Mugnai, "Solitary waves for nonlinear Klein-Gordon-Maxwell and Schrödinger-Maxwell equations," Proceedings of the Royal Society of Edinburgh, vol. 134, no. 5, pp. 893-906, 2004.

[18] D. Ruiz, "The Schrödinger-Poisson equation under the effect of a nonlinear local term," Journal of Functional Analysis, vol. 237, no. 2, pp. 655-674, 2006.

[19] L. Zhao and F. Zhao, "Positive solutions for SchrödingerPoisson equations with a critical exponent," Nonlinear Analysis: Theory, Methods and Applications, vol. 70, no. 6, pp. 2150-2164, 2009.

[20] C. Miranda, "Unosservazione su un teorema di Brouwer," Bollettino dell Unione Matematica Italiana, vol. 3, pp. 5-7, 1940.

[21] W. A. Strauss, "Existence of solitary waves in higher dimensions," Communications in Mathematical Physics, vol. 55, no. 2, pp. 149-162, 1977.

[22] M. Willem, Minimax Theorems, Birkhäuser, Boston, 1996. 